\title{
Bilderwelt, Lebensordnung und die Rolle des Betrachters im antiken Griechenland
}

This article addresses the question what role sculptures played in the social life of ancient Greece. Sculptures met social and cultural needs in specific situations; a close relationship existed between their functions and their installation in diverse living spaces in accordance with various norms, usages, rules and laws. The role of sculptures consisted in lending a permanent presence to events and personages that were far removed temporally and spatially. Human interaction with sculptures took place not in a »museal habitus« of interpretive contemplation exclusively within the confines of »art« or »culture; « rather, it consisted in a participatory »life with images.« In a social context, sculptures enjoyed, along with other environmental elements, personages and events, a greater or lesser degree of attention depending on their significance in a given situation. Of particular interest is the noteworthy fact that although ancient, especially Greek, sculptures were often set up in clearly visible locations, they could also be installed with surprisingly little consideration for the viewer. Sculptures constituted, along with other cultural elements, an ordered world (kosmos) in which mankind established and found its bearings, but a world which, independent of the rules of optimal visibility, possessed a certain degree of autonomy.

\section{Räume von Bildwerken in der Antike und heute}

Der römische Architekt und Architekturtheoretiker Vitruv mokiert sich über die Bewohner von Alabanda, einer Provinzstadt im südwestlichen Kleinasien: Sie hätten auf der Agora Standbilder von Diskuswerfern, Läufern und Ballspielern, in ihren Sportstätten dagegen Statuen von Rechtsanwälten stehen ${ }^{1}$. Die Maßstäbe, von denen er dabei ausgeht, werden im 4. Jh. v. Chr. von dem athenischen Staatsmann Lykurg bestätigt, der voller Stolz sagt, auf der Agora seiner Stadt seien ausschließlich Standbilder von Feldherren und den Tyrannenmördern, nicht aber von Athleten zu sehen ${ }^{2}$. Es gab also Standards, die definierten, welche Art von Bildwerken für welche Orte und Lebensräume angemessen war. Dabei müssen wir uns freilich hüten, Vitruvs Maßstäbe absolut zu setzen. Denn natürlich hatten die Leute von Alabanda ebenfalls ihre Gründe für ihre abweichende Praxis: Sie folgten nur anderen Maßstäben. Während in Athen und Rom die Agora bzw. das Forum bewusst als ein politischer Raum konzipiert wurde, schloss die Stadt Alabanda die athletischen Traditionen Griechenlands in die Gestaltung ihres öffentlichen Zentrums ein.

Die Griechen und Römer lebten, vielleicht mehr als alle anderen Kulturen der Weltgeschichte, mit Bildwerken. Alle Bildwerke hatten ihren Ort in sozialen Räumen. Die Räume

Eine erste Version dieses Textes habe ich im Juli 2007 als Herbert Lutz Gedächtnis Vorlesung an der Universität München, eine überarbeitete Version im Januar 2008 als Abschiedsvorlesung an der Universität Heidelberg vorgetragen. In weiterem Zusammenhang wird dies Thema wieder aufgenommen in meinem Buch »Visual Power in Ancient Greece and Rome«, Sather Lectures 94, 2007 (in Vorbereitung).

$1 \quad$ Vitruv 7, 5, 6 (Tadel aus dem Mund des Mathematikers Lycinus).

$2 \quad$ Lykurg. Leocrates 51. 
waren dafür maßgebend, welche Bildwerke in ihnen aufgestellt wurden, und umgekehrt prägten die Bildwerke den Charakter der Räume. Räume und Bildwerke definierten sich wechselseitig.

Für den Historiker der antiken Kunst bedeutet diese Situation eine Herausforderung ersten Ranges. Denn sie steht im diametralen Gegensatz zu den Bedingungen und Institutionen des neuzeitlichen Konzepts von >Kunst<. In unserer eigenen Vorstellung ist ein Bildwerk eine Schöpfung der >Kunst<, durch die ein >Künstler < einer subjektiven Vorstellung visuelle Form gibt. >Freie< Kreativität und Subjektivität sind Grundelemente dieses Konzepts des >Künstlers<. Sein Werk drückt durch seine kreativen Formen einen subjektiven >Sinn< aus, und die Betrachter sind aufgefordert, sich diesen >Sinn< des Werkes durch einen Akt des subjektiven Verstehens anzueignen.

Die spezifischen Räume dieses Konzepts der >Kunst< sind das Museum und das Buch. Das Museum ist eine Institution, die Kunstwerke und Betrachter in einem autonomen ästhetischen Raum vereinigt. Dort ist das Werk aus seinen ursprünglichen Kontexten und Funktionen herausgelöst und mit anderen Werken in neue ästhetische und historische Kontexte eingeordnet. Ähnlich ist der Betrachter aus dem Kontext seiner sozialen Welt herausgelöst und in eine Sphäre der >Kunst< versetzt. Dies ist ein Raum der reinen intensiven Betrachtung, entweder der intuitiven Wahrnehmung oder der intellektuellen Analyse. Das heißt: Das Museum ist ein Raum einer Haltung, die man als >musealen Habitus< bezeichnen kann.

Das Buch mit seinen Räumen der Produktion und Rezeption, Schreibtisch und Bibliothek, ist ein Laboratorium, in dem Autoren und Leser die Werke der Kunst nach wissenschaftlichen Kategorien wahrzunehmen und zu interpretieren suchen. Auch hier werden die Kunstwerke in neue Kontexte eingeführt: Sie werden zu Beispielen für die Geschichte der Stile, für die Ordnung der Ikonographie, für die Grammatik der Bildsprache gemacht.

Aus heutiger Sicht besitzen diese Kategorien ein großes Potential an historischer Erklärung; sie scheinen uns so evident, dass wir Kunstwerke kaum mehr anders zu sehen vermögen. Wir müssen uns aber klar machen, dass dies moderne Konstrukte sind, die wenig damit zu tun haben, warum die Bildwerke in der Antike geschaffen und aufgestellt und wie sie wahrgenommen wurden. Kein antikes Bildwerk wurde geschaffen, um ein Schritt in der Stilgeschichte, ein Beispiel einer Ikonographie oder ein Element der Bildsprache zu sein.

Das heißt: Ein angemessener Zugang zu den Werken der antiken Kunst muss zunächst vor allem zum Ziel haben, die Aufstellung und die Funktionen der Bildwerke an den Orten herauszufinden, für die sie bestimmt waren. Darüber hinaus aber stellt sich die Aufgabe, die Praxis und die sozialen Lebensformen zu rekonstruieren, die die Wahrnehmung von Bildwerken und den Umgang mit ihnen bestimmten³.

3 Pionierarbeit in dieser Richtung: Stemmer 1995. Grundsätzlich wichtig: Gell 1998. Dazu Osborne Tanner 2007. 


\section{Die Praxis der Aufstellung von Bildwerken}

Im antiken Griechenland hatten alle Bildwerke, in Skulptur, Malerei und anderen Medien, klar bestimmte Funktionen: als Kultbilder in den Tempeln, Votivgaben in den Heiligtümern, Bildnisse der Toten auf den Gräbern, politische Denkmäler und Ehrenstatuen auf öffentlichen Plätzen, Ausstattung privater Wohnsitze, Geräte und Gefäße für Ritual und Lebensvollzug und so fort. Für all diese Bereiche aber gab es bestimmte Praktiken, Regeln und Normen, die die Herstellung, den Gebrauch und die Wahrnehmung der Bildwerke prägten ${ }^{4}$. Zwei Beispiele sollen diese Praxis und ihre Folgen erläutern: die Standbilder von siegreichen Athleten und die Bildnisstatuen von Staatsmännern.

\subsection{Athletenstatuen}

Die ersten berühmten Personen, die in Griechenland mit öffentlichen Bildnisstatuen geehrt wurden, waren Sieger in den gesamtgriechischen Spielen. Für ihren Ruhm gab es zwei Bühnen: zum einen die großen Heiligtümer, in denen sie den Sieg errungen hatten, vor allem Olympia, zum anderen ihre Heimatstädte5.

In den Heiligtümern entwickelte sich die Errichtung von Athletenstatuen im Rahmen des religiösen Weihgeschenks: als Gabe an die Gottheit zum Dank für den Sieg. Dabei kamen zwei Tendenzen zur Geltung, die umso mehr divergierten, je höher die Ansprüche der Sieger auf öffentliches Prestige waren: Zum einen ging es um den Dank an die Gottheit, zum anderen um Ruhm für den Sieger selbst. Es ist bekannt, dass athletische Siege in den großen gesamtgriechischen Wettkämpfen außerordentliche gesellschaftliche Anerkennung eintrugen, weit über den Bereich der Athletik hinaus ${ }^{6}$. In diesem Sinn ist die Sitte der Aufstellung von Siegerstatuen zu verstehen: Standbilder von Siegern waren die beste Möglichkeit, diesen Ruhm über den Augenblick des Sieges hinaus $\mathrm{zu}$ verewigen.

Umso schärfer aber waren die Kontrollen, die dabei ausgeübt wurden: für die Zulassung zu den Wettkämpfen, die Einhaltung der Regeln, die Bestimmung der Sieger - und besonders für die Errichtung von Standbildern. In Olympia untersagten die offiziellen Beamten des Kults, die Hellanodiken, jede Form von überzogener Darstellung, sei es im Format, sei es in eklatanter Ähnlichkeit; sie waren weit strenger bei der Zustimmung zu Standbildern im Heiligtum als bei der Zulassung von Athleten zu den Kämpfen. Unter

4 Versuch einer Geschichte der griechischen Bildkunst im Rahmen ihrer sozialen Orte und Situationen:

T. Hölscher 2007 .

$5 \quad$ Allgemein zu griechischen Athletenstatuen: Rausa 1994; Smith 2007; Scott 2010, 153. 159-162.189-190. 196-201. 209-210. 213-217.

6 Mann 2001. 
diesen Voraussetzungen entwickelte sich in Olympia und sicher auch an anderen Orten eine vielfältige Praxis, die von der Spannung zwischen Dank an die Gottheit und Rühmung der Sieger geprägt ist?:

- Ein siegreicher Athlet konnte unmittelbar nach dem Sieg ein Standbild von sich aufstellen. Das war zunächst ein Akt der Dankbarkeit an die Gottheit. Wenn er mehr als einmal einen Sieg errungen hatte und jedes Mal eine Statue errichtete, trat der Aspekt der Selbstrühmung schon mehr in den Vordergrund ${ }^{8}$.

- Der Ruhm des Sieges war aber nicht nur eine persönliche Sache: Oft wurden Standbilder von athletischen Siegern von ihren Verwandten errichtet. Das konnte sowohl unmittelbar nach dem Sieg als auch in späterer Zeit, insbesondere durch den Sohn, geschehen. Daraus wird deutlich, welche Bedeutung athletische Siege für das Prestige der Familie hatten. Berühmte >Dynastien< von athletischen Siegern stellten ihre Bildnisse nebeneinander auf und bildeten damit große Familiengalerien 9.

- Schließlich konnte sogar die Gemeinschaft der Polis einem Sieger eine Statue in einem gesamtgriechischen Heiligtum errichten. Das geschah z. T. lange nach seinem Tod: So stellte Sparta im mittleren 5. Jh. in Olympia ein Standbild des Chionis auf, der zwei Jahrhunderte früher dreimal hintereinander gesiegt hatte, zur selben Zeit als dieselbe Stadt dies Heiligtum auch sonst zur Bühne ihrer politischen Ansprüche machte $^{10}$.

In Olympia lässt sich auch ungefähr die Aufstellung der Athletenstatuen erkennen ${ }^{11}$. Sie wurden vor allem entlang den Wegen der großen Rituale im Heiligtum errichtet: an dem Weg der großen Prozession vom Eingang im Süden zu dem großen Altar und an den Wegstrecken um den Tempel des Zeus, an dessen Rückseite der heilige Ölbaum stand, von dem die Zweige für die Siegerkränze geschnitten wurden. Die Standbilder der früheren Sieger nahmen also an den gemeinsamen Auftritten der Festgemeinschaft teil, als ideale Zuschauer und zugleich als leuchtende Exempel für die lebenden Teilnehmer.

Daneben konnte die Form der Siegerstatue von den Siegern selbst in ihrer eigenen Stadt eingesetzt werden, um ihre politische Stellung zu stärken. In Athen mobilisierte sich offenbar die konservative Faktion um die Mitte des 5. Jhs. gegen Perikles, indem sie ihren athletischen Ruhm auf der Akropolis durch aufsehenerregende Standbilder in

7 Zum Folgenden s. besonders: Amandry 1957; ferner Gross 1969. - Zu den Hellanodiken: Lukian. Imagines 11.

8 Weihung unmittelbar nach dem Sieg: Amandry 1957, 63-64, nach mehrfachen Siegen: 64-66.

9 Aufstellung durch Verwandte, gewöhnlich den Sohn: Amandry 1957, 64-67. - In späterer Zeit: Amandry 1957. - Zusammenstellung zu >Galerien<: am berühmtesten die Gruppe der Diagoriden von Rhodos: Paus. 6, 5, 1- 6, 7, 1. Amandry 1957, 67.

10 Paus. 6, 13, 2. Datierung um die Mitte des 5. Jh. durch den ausführenden Bildhauer Myron. Weitere Fälle: Amandry 1957, 67.

11 s. dazu Herrmann 1988. 
Erinnerung brachte: Besonders spektakulär war ein gewisser Pronapes, der sich als Ankläger gegen Themistokles hervorgetan hatte und der seinen vornehmen Anspruch mit einem Denkmal kundtat, das ihn auf der Quadriga darstellte, und damit seine Siege im Wagenrennen feierte ${ }^{12}$.

Die Praxis der Errichtung von Standbildern für siegreiche Athleten war also vielfältig, sie war gesteuert von klar erkennbaren religiösen Motiven und politischen Zielen.

\section{2 Öffentliche Ehrenstatuen}

Der nächste Schritt, mit dem herausragenden Bürgern öffentliches Ansehen und gesellschaftliches Prestige verliehen wurde, war die Errichtung öffentlicher Ehrenbildnisse. Diese Praxis entwickelte sich im 5. Jh. v. Chr. und erhielt in Athen im 4. Jh. eine relativ standardisierte Form. Ehrenstatuen garantierten den dargestellten Personen Präsenz und Gedächtnis im öffentlichen Raum. In den hoch kompetitiven Gesellschaften der griechischen Stadtstaaten waren solche Auszeichnungen wirksame Mittel zur Ausbildung von Macht. Aus diesem Grund waren Ehrenstatuen Gegenstände von heißen politischen Debatten: Erst in der Volksversammlung, wo die Entscheidungen über die Errichtung gefällt wurden; später in öffentlichen Diskursen und politischen Diskussionen, in denen aus unterschiedlichsten politischen Positionen Urteile geäußert und Kommentare abgegeben wurden. Diese Diskurse waren der Grund dafür, dass die Praxis der Errichtung von öffentlichen Ehrenstatuen immer mehr in kollektiv akzeptierten Formen geregelt wurde: Die Gefahr von Konflikten wurde durch Regulierung und Ausrichtung an Präzedenzfällen eingeschränkt ${ }^{13}$.

Die entscheidenden Fragen, um die es in solchen Debatten ging, waren: Wer sollte mit einem öffentlichen Ehrenbildnis ausgezeichnet werden? Wer sollte die Ehrung durch eine Bildnisstatue vornehmen? Wann war der angemessene Zeitpunkt für eine solche Ehrung, zu Lebzeiten oder erst nach dem Tod? Wo sollte ein Ehrenbildnis errichtet werden? Aus welchen Gründen, d. h. für welche Leistungen und Verdienste war eine öffentliche Ehrenstatue gerechtfertigt? Und schließlich: Wie und in welchen Formen sollten die Bildnisse die Ehrung zum Ausdruck bringen ${ }^{14}$ ?

\footnotetext{
12 Zu den Athletenstatuen auf der Akropolis von Athen s. Raubitschek 1939, 155-160; Raubitschek 1949, 464; Keesling 2003, 170-175. Pronapes: Krumeich 1997, 113-114. Weitere Athletenstatuen dieser Zeit von Staatsmännern der aristokratischen Faktion, Kallias Didymiou: Krumeich 1997, 89-91; Kallias Hipponikou: Krumeich 1997, 91-93. Zu der hier vertretenen politischen Deutung s. Raubitschek 1939, 164. Die Tendenz von Krumeich, bei diesen Bildwerken eine rein religiöse Motivation zu erkennen und politische Ziele weitgehend auszuschließen (zusammenfassend Krumeich 1997, 214-215), scheint mir nicht zutreffend zu sein; dazu an anderem Ort.

13 Gründliche Untersuchung der Zeugnisse bei Krumeich 1997, passim. Zur Praxis in Athen s. bereits Thompson - Wycherley 1972, 155-160. Dazu Tanner 1992; Krumeich 1997, 207-212; Dillon 2006, 101-106; Tanner $2006,97-140$.

14 Dazu besonders die Schriftquellen, in Ausschnitten gesammelt bei Wycherley 1957, 207-217. Wichtig ist dabei immer der weitere Kontext in den literarischen Werken.
} 
Die Entstehung öffentlicher Ehrenstatuen in Griechenland kann mit zwei Beispielen beschrieben werden, die den Anfangs- und einen ersten Endpunkt bezeichnen. Um 540 v. Chr. weihte ein gewisser Aiakes, Sohn des Brychon, auf Samos ein überlebensgroßes Marmorbildnis von sich selbst, auf einem Thron sitzend, der Göttin Hera, wohl in ihrem städtischen Heiligtum. Mit einiger Wahrscheinlichkeit kann er als Vater des Tyrannen Polykrates identifiziert werden, jedenfalls muss er einer der führenden Männer von Samos gewesen $\operatorname{sein}^{15}$. Ein Bildnis von sich selbst einer Gottheit zu weihen, war damals eine geläufige religiöse Praxis; nur der Typus einer thronenden Gestalt und das große Format bezeugen Aiakes' außergewöhnliche politische Ambitionen ${ }^{16}$. - Im Jahr 394 v. Chr. wurde der athenische Feldherr und Staatsmann Konon von der Volksversammlung seiner Stadt mit einer Reihe hoher Auszeichnungen geehrt. Die größte dieser Ehren war eine Bildnisstatue auf der Agora. Ein bekannter Kopftypus eines Strategen mit Helm wurde gelegentlich als Konon gedeutet; die Gründe dafür sind nicht ausreichend, aber ungefähr in dieser Weise kann man sich den Kopf des Standbildes vorstellen. Der Körper könnte nackt gewesen sein, wie bei der Bronzestatuette eines Strategen, die wohl ein großformatiges Original dieser Zeit wiedergibt. Wie dem auch sei, hier war es die institutionelle Autorität des athenischen Staates, der diese öffentlich-politische Anerkennung im Namen der gesamten Gemeinschaft der Bürger verlieh ${ }^{17}$.

Zwischen diesen beiden Polen wurde die Verherrlichung politischer Leistung und Macht immer deutlicher vorangetrieben. Ein entscheidender Schritt war die Statuengruppe der Tyrannenmörder Aristogeiton und Harmodios. Sie war von der Gemeinschaft der Bürger errichtet worden, im politischen Zentrum von Athen, frei von jeder religiösen Funktion; dort diente sie einzig der Rühmung einer politischen Tat, die der Gründungsmythos der athenischen Demokratie werden sollte. In diesem Sinn standen die Statuen der Tyrannenmörder als Vorbild am Rand der Stätte der demokratischen Volksversammlung, die ihr politisches Grundziel in der Abwehr einer neuen Tyrannis sah. Bei den politischen

15 Freyer-Schauenburg 1974, 139-146 Nr. 67.

16 Zur Praxis der Selbstweihung s. die wichtige Studie von Himmelmann 2001. Himmelmann sieht hierin eine entscheidende, religiöse Wurzel der griechischen Porträtstatue, nicht zuletzt auch der realistischen Darstellungsweise mit individueller Physiognomie. Dagegen sehe ich den entscheidenden Schritt in Richtung auf eine politische Bedeutung gerade im Abgehen von dieser Praxis in Form der Statuensetzung durch die politische Gemeinschaft. Die Entwicklung individueller Bildnisformen vollzieht sich nicht Schritt für Schritt parallel zu dieser Politisierung, sondern steht in einer signifikanten Spannung zu ihr. Aber im Wesentlichen dienen diese Formen meines Erachtens der Darstellung - und Anerkennung! - einzigartiger Rollen im öffentlichen Leben. Das kann hier nicht genauer ausgeführt werden.

17 Bildnis des Konon: Krumeich 1997, 207-208. - Strategen-Typus >Pastoret<: Pandermalis 1969, 46-55. Wegen der Zahl der römischen Kopien offenbar ein in späterer Zeit berühmter Mann. Eine Alternative innerhalb des Zeitraums, der nach dem Stil möglich scheint, wäre Alkibiades; bei ihm möchte man allerdings mehr jugendliches Charisma erwarten. - Bronzestatuette Hartford: Bielefeld 1962. - Anders Himmelmann 1990, 86-101, der für die Darstellung der Strategen Kleidung annimmt (und die Statuette Hartfort für ein klassizistisches Bild des Ares hält). 
Entscheidungen sollte jeder Bürger ein potentieller Tyrannenmörder werden. Wenn man sich vor Augen hält, dass durchaus noch eine große Gruppe von einflussreichen Anhängern der Tyrannen in Athen lebte, so war die Besetzung der Agora durch ein Denkmal der Attentäter ein Akt von höchster Brisanz: Es war eine symbolische Vertreibung der Gegner aus dem politischen Zentrum der Stadt. Die Tyrannenmörder waren das erste eigentlich >politische Denkmal< Griechenlands ${ }^{18}$.

Die Entstehung des politischen Denkmals ist das eindeutigste Symptom für das, was Christian Meier die »Entstehung des Politischen « genannt hat ${ }^{19}$. Mit dem politischen Denkmal wird der öffentliche Raum der Agora in einer neuen Weise als >politischer Raum< definiert.

Die Personen, die mit öffentlichen Ehrenstatuen ausgezeichnet wurden, waren zunächst ausschließlich erfolgreiche Feldherren, wie Konon, der Athen durch einen Seesieg von der Vorherrschaft Spartas befreit hatte. Spätere Ausweitungen der Ehre waren heftig umstritten: So wurden einflussreiche politische Redner wie Aischines oder Demosthenes erst postum mit öffentlichen Bildnisstatuen geehrt ${ }^{2 \circ}$. Schließlich wurden auch auswärtige Wohltäter des Staates einbezogen: Zuerst der zyprische König Euagoras, weil er Konon gegen Sparta unterstützt hatte; später drei Könige vom Bosporos, Pairisades, Satyros und Gorgippos, weil sie Athen mit Kornlieferungen unterstützt hatten ${ }^{21}$.

Dabei gab es eine Hierarchie der Orte. Mehrfach wurde beschlossen, die Wahl des Standortes den Stiftern oder dem Geehrten zu überlassen. Dabei werden sie eine möglichst wirkungsvolle Aufstellung gewählt haben; in späteren Inschriften heißt es oft, Standbilder für besonders prominente Männer sollten »an dem sichtbarsten Ort« errichtet wer$\operatorname{den}^{22}$. Eine zusätzliche Auszeichnung lag in der Aufstellung in Verbindung mit anderen berühmten Monumenten. So wurden die Standbilder des Konon und des Euagoras vor der Halle des Zeus Eleutherios errichtet, des Gottes der Freiheit, der Athen vor den Persern und anderen Unterdrückern gerettet hatte: ein angemessener Platz für die Männer, die Athens Unabhängigkeit von Sparta erkämpft hatten. Dass der Feldherr Timotheos dann neben seinem Vater Konon aufgestellt wurde, ist ein frühes Beispiel für die sukzessive Entstehung von >Familiengalerien<. Ähnlich wurde eine Bildnisstatue des Kallias, der die berühmte >Friedens«-Abmachung mit den Persern ausgehandelt hatte, lange nach seinem Tod neben dem Altar und dem Standbild der Friedensgöttin Eirene aufgestellt ${ }^{23}$. Und so fort.

18 Brunnsåker 1971; Fehr 1984; Taylor 1992; F. Hölscher 2010.

19 Meier 1980.

20 Aischines: Richter 1965, II 212-215; Zanker 1995, 51-54. - Demosthenes: Richter 1965, II 215-223; Zanker 1995, 85-89.

21 Euagoras: s. o. Anm. 17 zu Konon. - Herrscher vom Bosporos: Deinarch. Demosthenes 43. IG II ${ }^{2}$ 653, 40-42. Wycherley 1957, n. 700. 711.

22 Wahl des Standortes: Wycherley 1957, Nr. 278; vgl. 701. 704. - Epiphanestatos topos: Bielfeldt 2012.

23 Konon: s. o. Anm. 17. - Timotheos: Aischin.Ctes. 243. Cornelius Nepos, Timotheus 2, 3. - Kallias: Paus. 1, 8, 2. Dazu Simon 1988, 63-64; Krumeich 1997, 93. 
Sogar mit Gesetzen griff man ein. Es war verboten, Ehrenstatuen neben den Tyrannenmördern aufzustellen, weil keine andere Person mit diesen Gründerheroen des Staates auf eine Stufe gestellt werden sollte. Nur zwei Ausnahmen wurden gemacht, eben um diesen Vergleich doch zu suggerieren, für die makedonischen Herrscher Antigonos und Demetrios Poliorketes nach der Befreiung Athens von der makedonischen Vorherrschaft, dann für Brutus und Cassius nach dem Mord an Julius Caesar. Diese Taten wurden als Befreiung von Tyrannis gedeutet, vergleichbar der Tat der Tyrannenmörder ${ }^{24}$.

Schließlich wurde eine sehr konkrete Unterscheidung bei der Finanzierung gemacht. Bei prominenten Männern übernahm selbstverständlich der Staat die Kosten für die Ehrenstatue. Bei weniger bedeutenden Personen aber konnte es auch vorkommen, dass die Volksversammlung nur die Erlaubnis für eine Ehrenstatue aussprach, die der Geehrte dann selbst bezahlen sollte ${ }^{25}$.

Dies waren die Anfänge einer differenzierten Praxis der Errichtung öffentlicher Ehrenstatuen, die dann in den folgenden Jahrhunderten bis in die römische Kaiserzeit weiter entwickelt und gepflegt wurde.

Wenn man diese Bildnisstatuen zusammen sah, so ergaben sie eine historische Physiognomie des athenischen Staates, mit seinen Gründerheroen, seinen führenden Staatsmännern und seinen auswärtigen Wohltätern.

Die Bildnisse siegreicher Athleten und ruhmreicher Staatsmänner bezeugen ein umfassendes Phänomen der griechischen Bildkultur. Bildwerke verschiedenster Art stellten >Bilder-Welten< dar, die die Lebensräume der menschlichen Gesellschaften mit Sinn erfüllten. Das gilt nicht nur für die politischen Räume der Agora, sondern auch für die sakralen Räume der Heiligtümer, für die Gedenk-Räume der Nekropolen und für die privaten Räume des Wohnens. Darum noch ein Beispiel aus einem großen griechischen Heiligtum.

\subsection{Schatzhaus von >Sikyon<}

Im Apollon-Heiligtum von Delphi wurde eine Serie von Reliefs aus archaischer Zeit gefunden, die als Serie von Metopen ein Schatzhaus geschmückt haben müssen, d. h. einen kleinen Bau, der ein wertvolles Weihgeschenk einer Stadt barg; die Zuweisung an die Stadt Sikyon ist nicht sicher, aber wahrscheinlich. Auf diesen Reliefs sind Mythen dargestellt, die für die betreffende Stadt offenbar paradigmatische Bedeutung hatten ${ }^{26}$.

Zwei dieser Mythen stellen große kollektive Unternehmen dar, wie sie auch in der Lebenswelt der archaischen Zeit hoch geschätzt waren. Ein Bild eines Ebers stammt aus einer Schilderung der Jagd auf den Eber von Kalydon, zu der sich mythische Helden aus

24 Verbot: Thompson - Wycherley 1972, 159. - Antigonos und Demetrios: Diod. 20, 46, 2. - Brutus und Cassius: Cass.Dio 47, 20, 4.

25 Asandros (Makedone, Reiterstatue): IG II ${ }^{2}$ 450, b 7-12.

26 Knell 1990, 18-23; T. Hölscher 2009, 58-61. 
vielen Städten vereinigt hatten. Gleichzeitige Vasenbilder können die ursprüngliche Komposition veranschaulichen. Dieser Mythos konnte als Vorbild für die gegenwärtigen Gesellschaften dienen, so wie etwa verschiedene Städte sich im sog. Heiligen Krieg zum Schutz von Delphi gegen die Stadt Phokis zusammengeschlossen hatten. Da Jagd und Krieg in archaischer Zeit als eng verwandte Bereiche männlicher Bewährung angesehen wurden, konnte die kalydonische Jagd zum mythischen Muster gemeinsamer Kriegszüge werden. Dabei wird in den gleichförmigen Bewegungen der Jäger ein Ethos der koordinierten Aktionen, des Zusammenhalts und der Gleichheit vor Augen gestellt, das grundlegend für die archaischen Gesellschaften war.

Eine andere Metope schilderte die Fahrt des Schiffes Argo mit vielen Helden zum fernen Kolchis, wo sie das goldene Vlies rauben sollten. In diesem Mythos sind die Seefahrten kühner Adeliger in ferne Länder präfiguriert, bei denen sich Handel, Piraterie und Landgewinnung zu einem Ideal expansiver Kühnheit verbanden. Auch hier war der Gemeinschaftsgeist der mythischen Helden aus allen Teilen Griechenlands ein Vorbild für die kollektive Solidarität gegenwärtiger Seefahrer.

Weitere Metopen rühmten mythische Muster persönlichen Heldenmuts. Die Dioskuren Kastor und Polydeukes stehlen gemeinsam mit den Brüdern Idas und Lynkeus eine große Herde von Rindern. Auch hierin wird eine Tugend gepriesen, die in der Gegenwart der archaischen Zeit hoch im Kurs stand: Gewaltsamer Rinderraub war damals noch eine übliche Praxis, vor allem in den Grenzzonen zwischen den Stadtstaaten, wo immer wieder Konflikte zwischen reichen Herdenbesitzern sich zu Kriegen zwischen größeren politischen Gemeinschaften entwickelten. Der Raub von Rindern galt als Beweis von männlichem Mut, für den große Helden das Vorbild abgaben: Achill war der berühmteste individuelle Rinderräuber, die Brüderpaare auf den delphischen Reliefs tun dasselbe in koordinierter Solidarität.

Selbst der wilde Mythos von Zeus, der sich der schönen Königstochter Europa in Gestalt eines Stieres nähert, sie abschleppt und liebt, spricht ein zentrales Thema der archaischen Gesellschaft an: die Hochzeit und das Verhältnis zwischen den Geschlechtern. Das Grundkonzept der Hochzeit, das in diesem Mythos präfiguriert war, war der Raub der Braut durch den Bräutigam. Solche frühen rituellen Praktiken stehen hinter dem Mythos des Helden Peleus, der die Meeresgöttin Thetis im Ringkampf bändigt, so wie in der Lebenswelt die >wilden ₹ Züge junger Mädchen für ihre Rolle als Herrin des Hauses und Mutter der Kinder >gezähmt werden sollten. Im Mythos der Europa wird ein anderer Aspekt gezeigt: die Einwilligung einer schönen jungen Frau, sich von dem Stier davontragen zu lassen, der die höchste Virilität verkörpert und dabei Zeus, den mächtigsten aller Bräutigame, einschließt.

Der Bildschmuck dieses Schatzhauses ist weit mehr als eine Sammlung von sinteressanten< Mythen, er ist ein ganzer Kosmos von Grundkonzepten der archaischen Gesellschaften. Dieser Kosmos diente als ideeller Rahmen für das große verlorene Weihgeschenk an den Gott, für den der Bau errichtet worden war. Die Stadt, die das Schatzhaus stiftete, hat dafür keine spezifischen Mythen aus der eigenen Vergangenheit gewählt, sondern sich als Vertreterin und Protagonistin von mythischen Leitbildern präsentiert, 
die allgemeine Geltung besaßen. In diesem Sinn aber hat das Schatzhaus im Heiligtum von Delphi eine präzise Funktion: Es bewirkt die Präsenz der Stadt mit ihren zentralen Leitvorstellungen vor der gesamtgriechischen Öffentlichkeit in diesem Heiligtum, gewissermaßen als Verstetigung der vornehmen Gesandtschaften, die die Bürgerschaft bei den großen Festen repräsentierten.

In diesem Sinn sind alle figürlichen Weihgeschenke, in großen wie in kleinen Formaten, in den Heiligtümern zu verstehen. Sie alle sind Teil eines vielfältigen Zusammenspiels von Bildwerken, die die verschiedensten Stifter errichteten und die mehr und mehr zu einer komplexen Bilderwelt zusammenwuchsen. Das Spektrum der Möglichkeiten ist weit und es änderte sich von Epoche zu Epoche. Zur selben Zeit, als das Schatzhaus von >Sikyon< gebaut wurde, stellte die Stadt Argos in Delphi Standbilder ihrer Helden Kleobis und Biton auf, die ein leuchtendes Exempel der Sohnesliebe dargestellt hatten: Hier präsentierte sich eine Stadt mit ihren eigenen Vertretern als Muster für ganz Griechenland. Die Stadt Naxos dagegen errichtete eine hohe Säule mit einer Sphinx, die als unheimliches Wesen der wilden Natur dies Heiligtum als Ort der göttlichen Ordnung bewachte ${ }^{27}$. Individueller Ruhm, allgemeine Leitbilder und göttlich-mythische Machtwesen ergaben einen ständig sich wandelnden und erweiternden religiösen Raum, in dem die lebenden Menschen die Rituale ihrer Lebensordnung vollzogen.

Dies ist ein Grundphänomen der antiken Bildkunst. Alle Bildwerke im antiken Griechenland und Rom, die Kultstatuen in den Tempeln, die Weihgeschenke in den Heiligtümern, die Bilder auf den Gräbern und so fort, erfüllten klar bestimmbare Funktionen, an bestimmten Orten und in bestimmten Situationen des sozialen Lebens. Überall gab es Gebräuche und Normen, Regeln und Vorschriften, die die Praxis der Aufstellung und den Umgang mit den Bildwerken bestimmten.

\section{Die Rolle von Bildwerken im gesellschaftlichen Leben}

Wie konnten die Bildwerke diese Funktion im gemeinschaftlichen Leben erfüllen? Der eigentliche Sinn eines Bildwerks in der Antike war: >präsent< zu machen. Ein Bild hat die Kraft, Personen und Gegenstände aus der Distanz des Raumes und der Zeit in die Welt der lebenden Menschen zu versetzen ${ }^{28}$.

Diese Kraft, etwas unmittelbar vor Augen zu führen, ist die wichtigste Leistung von Bildern gegenüber den in anderer Hinsicht überlegenen Texten. Dass sie nicht nur für die Antike gilt, kann ein Vorgang aus unserer eigenen Zeit belegen. Vor einigen Jahren fiel die Prinzessin Caroline von Monaco vom Pferd und erregte damit eine Flut von

27 Kleobis und Biton: Rolley 1994, 168-170; Bol 2002, 143-145 Abb. 212 a-d (D. Kreikenbom). - Sphinx der Naxier: Amandry 1953, 1-32; Bol 2002, 169 Abb. 244 a-c (D. Kreikenbom).

28 Zum Folgenden s. allgemein: Niemeyer 1996; Steiner 2001. 
Medienberichten. Daraufhin verklagte sie mehrere Zeitschriften wegen »Verletzung ihrer äußeren Privatsphäre«. Da der Fall internationale Dimensionen annahm, ging er in verschiedenen Ländern bis in die höchsten Gerichtshöfe. In Deutschland entschied man eindeutig: Caroline sei eine Person von öffentlichem Interesse, daher sei die Berichterstattung zulässig. Differenzierter urteilte der Europäische Gerichtshof für Menschenrechte: Geschriebene Reports seien zuzulassen, aber Photographien bedürften der Zustimmung der betroffenen Person. Offenbar waren die Richter der Meinung, dass Bilder ein höheres Potential der Vergegenwärtigung und damit auch der Verletzung besäßen als Texte ${ }^{29}$.

In der Antike wurden die Götter, die in weiter Ferne lebten, durch Bildwerke in den Tempeln und in den Lebensräumen der Menschen >präsent< gemacht. Mythische Heroen und verstorbene Vorfahren wurden über die Distanz der Zeit in die Welt der Gegenwart versetzt. Zeitgenössische Personen von öffentlicher Bedeutung wurden in den städtischen Zentren in Bildnisstatuen vor Augen gestellt, wo sie in corpore nicht immer gegenwärtig sein konnten. Die Bildwerke stellten eine ideale Gemeinschaft von Göttern und Heroen, verstorbenen und lebenden Menschen dar, innerhalb derer die gegenwärtige Gesellschaft ihre kulturelle und ethische Orientierung definieren und ihre Lebenspraxis ausrichten konnte.

Diese Kraft der Bilder, >Präsenz $<$ zu schaffen, beruht auf ihrer erstaunlichen Lebendigkeit. Mit Bildwerken ging man um wie mit lebendigen Wesen. Das gilt zunächst für die Bilder von Göttern, über die nur wenige bekannte Züge in Erinnerung zu rufen sind. Die Kultbilder aus den Tempeln wurden z. T. in Prozessionen herumgetragen, in Flüssen und an der Meeresküste rituell gewaschen, mit Duftölen gesalbt, mit Kleidern angetan und mit Schmuck ausgestattet, als wären sie die Gottheit selbst. Von Götterbildern wurde berichtet, dass sie die Köpfe bewegt, Tränen geweint oder Blut geschwitzt und damit Zustimmung oder Abweisung in bestimmten Situationen kund getan hätten. Solche Praktiken und Vorstellungen sind nicht auf eine urtümliche Frühzeit beschränkt, sondern blieben bis in späte Zeiten lebendig. Die Epheser schickten ein Bild ihrer Göttin Artemis als Gesandte nach Rom zum Kaiser Caracalla, um ihm eine Bitte vorzutragen. Die bekannte Geschichte von einem jungen Mann, der sich in das Bild der Aphrodite von Praxiteles verliebte und sich nachts mit ihr in ihrem Tempel einschließen ließ, ist nur eines von vielen Beispielen eines weit verbreiteten $\operatorname{Topos}^{30}$.

Der mythische Prototyp dieser Vorstellungen ist der Ur-Bildhauer Daidalos, dessen Bildwerke angeblich gefesselt werden mussten, damit sie nicht davonliefen. Umgekehrt, und noch erstaunlicher, ist eine Stelle bei Euripides, wo die Troianerkönigin Hekabe Agamemnon um Gnade anfleht und sich wünscht, in ihren Armen, Händen, Haaren und dem Gang ihrer Füße, eine Stimme zu haben, »durch die Kunst des Daidalos oder eines

29 Stürner 2005.

30 Allgemein: Scheer 2000, 44-148; Bettinetti 2001; Steiner 2001; F. Hölscher 2004; F. Hölscher 2005; Chaniotis in Vorbereitung. - Artemis von Ephesos als Gesandte zu Caracalla: SEG XXXI 955. - Aphrodite von Knidos: Ps-Lukian, Amores 11-16. Dazu allgemein Olmos 1992. 
Gottes«, dass sie alle zusammen weinend seine Knie umfassten und ihm mit vielfältigen Worten ihre Klage vortrügen. Während also die Bildwerke des Daidalos voll wunderbaren Lebens sind, wird hier sogar umgekehrt die Lebendigkeit einer wirklichen Person mit der Ähnlichkeit zu einem Bildwerk des Künstlers beschworen ${ }^{31}$.

Ähnlich >lebend sind Bildwerke von menschlichen Wesen. Noch aus dem 5. Jh. v. Chr. wird berichtet, der berühmte Athlet Theagenes von Thasos habe einen persönlichen Feind gehabt, der sich nach seinem Tod an ihm rächte, indem er seine öffentliche Bildnisstatue auf der Agora täglich auspeitschte - bis das Bildwerk zurückschlug, auf ihn fiel und ihn tötete. Die Söhne des Opfers erhoben Anklage gegen das Bildwerk wegen Mordes, sie bekamen Recht und die Thasier warfen das Standbild zur Strafe ins Meer. Bald danach wurden sie von einer Hungersnot heimgesucht, woraufhin sie das Orakel in Delphi befragten und die Antwort erhielten, sie sollten alle Exilierten in die Stadt zurückholen. Das taten sie, doch die Hungersnot wiederholte sich. Auf eine neue Anfrage kam aus Delphi die Antwort, sie hätten Theagenes vergessen. Daraufhin fischten sie dessen Standbild aus dem Meer und stellten es wieder auf. Sie verehrten Theagenes' Bild über Jahrhunderte und der Held dankte ihnen mit wirkungsvollen Heilwundern. Das ständige Hin und Her zwischen Wirklichkeit und Bildwerk bezeugt eindrucksvoll, dass die Person und ihr Bild austauschbar waren ${ }^{32}$.

Im Jahr 480 v. Chr. verurteilten die Athener Hipparch, den Sohn des Charmos, einen alten entschiedenen Gefolgsmann der inzwischen vertriebenen Tyrannen, in Abwesenheit zum Tod. Da sie seiner nicht habhaft werden konnten, vollzogen sie die Strafe, indem sie sein bronzenes Bildnis auf der Akropolis einschmolzen. Aus der Bronze fertigten sie eine Stele an, auf der sie alle politischen Verräter aufschrieben und auf der Akropolis bekannt machten ${ }^{33}$. Das Bild war getötet worden, aber das Material schloss immer noch die Identität der Person ein und wurde darum mit den Namen derer gebrandmarkt, die seine politische Position vertraten. Sogar tote Gegenstände konnten in dieser Weise behandelt werden: Der archaische Gesetzgeber Drakon soll nicht nur Mörder aus Athen verbannt haben, sondern sogar Gegenstände, mit denen eine Person getötet worden war, aus dem Land verwiesen haben ${ }^{34}$.

Es kam vor, dass Bilder von Menschen hergestellt wurden, um Interaktion mit ihnen möglich zu machen. Als der Spartanerkönig Leonidas bei den Thermopylen fiel, konnte sein Leichnam nicht nach Sparta transportiert werden; darum fertigte man ein eidolon von ihm an, um mit ihm die Rituale des Begräbnisses zu vollziehen ${ }^{35}$. Zwei Jahrzehnte später verurteilten die Spartaner ihren Regenten Pausanias, den Feldherrn der Schlacht

31 Daidalos: Morris 1992, 215-237; Eur.Hec 836-840.

32 Paus. 6, 11, 2-9. Standort des Bildwerks auf der Agora von Thasos: Grandjean - Salviat 2000, 76-76 (mit

Lit.). Allgemein wichtig zu dem ganzen Fragenkomplex: Niemeyer 1996.

33 Lykurg. Leokrates 117-119. Krumeich 1997, 63-64.

34 Aischin.Ctes. 244; Paus. 1, 28, 11.

35 Hdt. 6, 58. Niemeyer 1996, 36. 
bei Plataiai, wegen Verrats zum Tod. Er floh in das Heiligtum der Athena, wo er im Schutz der Gottheit nicht verhaftet werden konnte; worauf seine Mitbürger ihn Hungers sterben ließen. Als daraufhin eine Seuche ausbrach, befahl das Orakel von Delphi den Spartanern, sie sollten zum Ersatz für den einen Menschen zwei Standbilder von ihm errichten. Bezeichnenderweise wird der Begriff soma gebraucht, der sowohl den Körper des Toten als auch das plastische Bildwerk bezeichnen kann ${ }^{36}$.

Ähnliche Vorstellungen waren in Kyrene in Geltung, wo ein Gesetz vorschrieb, wie man sich verhalten sollte, wenn man einen Flüchtling aufnehmen wollte, der aus einer anderen Stadt verbannt worden war. Um Frieden mit den Initiatoren der Verbannungen zu machen, sollte man Bildnisse von ihnen aus Holz oder Ton anfertigen, diese Bildnisse in das eigene Haus laden und sie mit Speise und Trank bewirten ${ }^{37}$. Weniger urtümlich, aber besonders rührend ist die Anekdote von einem athenischen Söldner, der beim Aufbruch in den Krieg sein Vermögen in die Hände der Ehrenstatue des Demosthenes auf der Agora legte - und es bei der Rückkehr unberührt vorfand. Es kommt nicht darauf an, ob die Geschichte wahr ist oder nicht: Jedenfalls wurde die bekannte Integrität des Demosthenes ohne Zögern auf sein Standbild übertragen und damit eine wirkungsvolle Schutzkraft für reales Geld geschaffen ${ }^{3}$.

In diesem Sinn bewirken Bildwerke für solche Personen eine machtvolle physische >Präsenz‘, die tatsächlich in Raum und Zeit weit entfernt waren, denen aber eine >konzeptuelle Präsenz gegeben werden sollte.

Diese >lebenden< Bildwerke waren bei allen Gelegenheiten und Situationen des öffentlichen Lebens gegenwärtig. In Olympia säumten die Standbilder früherer athletischer Sieger als >Betrachter und zugleich Vorbilder die Wege der Prozessionen und anderer Rituale. In Epidauros war der Platz des Altars vor dem Tempel von Standbildern gesäumt, deren Fundamente und Postamente sich erhalten haben. Damit wurde ein sakraler Raum abgegrenzt, in dem die Festgemeinde an dem Opfer teilnahm; diese Teilnehmer wurden wiederum umgeben von den Bildnissen berühmter Personen der Vergangenheit und Gegenwart, die dort eine ideale Gemeinschaft von Zuschauern bildeten. Noch eindrucksvoller ist die Situation an der Agora von Priene: Sie wurde an der Nordseite von der Prozessionsstraße durchquert; diese Straße wurde auf der einen Seite von einer treppenartigen Tribüne gesäumt, auf der anderen Seite von einer freien Zone, wo die Zuschauer der Prozessionen sich postieren konnten; und hinter ihnen erhob sich die Reihe der Ehrenstatuen bekannter Bürger, die im Bild an den Ritualen teilnahmen. Immer war da eine dichte Reihe von Bildern >großer Männer<, von Vorfahren und Vorbildern, die die lebenden Menschen beobachteten und ihnen ihre Leitbilder aufdrängten 39 .

36 Thuk. 1, 134, 4; Paus. 3, 17, 7-9. Krumeich 1997, 156-159.

37 SEG IX 72. Niemeyer 1996, 38-39.

38 Plut.Dem. 30, 5-31, 2; s. o. Anm. 20.

39 Olympia: T. Hölscher 2002. Epidauros: Gruben 2001 Abb. 115. Allgemein ausgezeichnete Untersuchung zu Priene s. Bielfeldt 2012. 
Mit >konzeptueller Präsenz< ist gemeint, dass die Bildwerke nicht magische Evozierungen der dargestellten Wesen sind. Im Gegenteil, die Schriftquellen heben sehr ausdrücklich den künstlichen Charakter dieser >lebenden< Bildwerke hervor, sie betonen die Materialien, Gold oder Stein, und bezeugen die Urheberschaft berühmter Bildhauer. Es waren geschaffene Bilder mit den Komponenten des Materials und des Stils: Gerade darin - hier liegt die eigentliche Aufgabe der kunstgeschichtlichen Analyse - kommt die konzeptuelle >soziale< Bedeutung dieser Gestalten zum Ausdruck. Allerdings war dies eine Bedeutung mitten im Leben: Die Funktion der Bildwerke, den dargestellten Personen und Gegenständen eine konkrete visuelle und materielle Präsenz zu geben, sollte dazu führen, dass die gegenwärtige Gesellschaft mit ihnen in konkreten Handlungen und Ritualen umgehen konnte. In diesem Sinn wurden Ehrenstatuen auf öffentlichen Plätzen an Festtagen bekränzt und auf diese Weise in die Festgemeinschaft integriert. Die Standbilder der Tyrannenmörder auf der Athener Agora wurden in öffentlichen Debatten als ideale Muster konkreten politischen Verhaltens aufgerufen. Wie geläufig das Bildwerk als Vorbild für das reale Verhalten war, zeigt eine höchst wirkungsvolle Szene in Aristophanes' Komödie »Lysistrate«, in der die Frauen die Macht im Staat ergreifen wollen: Dort tritt der Anführer der Männer, die die öffentliche Ordnung gegen die rabiaten Frauen verteidigen wollen, mit dem erheiternden Versprechen auf, sich kämpfend neben Aristogeiton zu stellen und sogar dessen Kampfhaltung einzunehmen. Die Kehrseite der Vorbildlichkeit zeigt sich in einer Anklage-Rede des Lykurgos, der seinen Gegner Lysikles dafür beschimpft, dass er es wage, in persona auf der Agora aufzutreten, obwohl er in seiner Stadt eine Erinnerung von Schande und Ehrlosigkeit hinterlassen habe: Das ist sicher vor dem Hintergrund all der Ehrenstatuen zu sehen, die die Agora zu einem Platz politischer Tugenden machten; zu diesen Bildnisstatuen stand der lebende Lysikles in eklatantem Widerspruch ${ }^{40}$.

In diesem Sinn wurden Götter und mythische Heroen, Herrscher, Staatsmänner und Athleten, Dichter und Philosophen in den Räumen des Lebens >konzeptuell präsent< gemacht. Die Toten und die Lebenden, die Abwesenden und die Gegenwärtigen bildeten eine konzeptuelle Gemeinschaft.

Wenn man zusammenfassend versucht, diesen Umgang mit den Bildwerken soziologisch zu gliedern, dann lässt sich das nach drei Grundkategorien tun: Raum, Zeit und Handlung.

- Sozialer Raum. Bildwerke dienten zur Ausstattung der wichtigsten Orte und Räume des sozialen Lebens: Heiligtümer, öffentliche Plätze und Gebäude, Wohnsitze, Gräber. Allgemein gab es dabei keine festgelegten Zuordnungen zwischen Räumen und Bildern, sondern ein dynamisches Wechselverhältnis. Wie der Fall von Alabanda

40 Bekränzung von Bildwerken an Festtagen: SEG VIII 529; XVIII 953, Z. 63. 71-72; XLVIII 742 B 7 (für die Hinweise danke ich A. Chaniotis). - Tyrannenmörder als Beispiel: F. Hölscher 2010. - Aristoph.Lys. 631-634. Schande auf der Agora: Lykurg. Lysikles, Fr. XII 1. 
zeigt, kam es durchaus zu Varianten in der Ausstattung von öffentlichen Plätzen: Die Agora war ein Rahmen für Bildwerke von >öffentlicher< Bedeutung, aber sie legte nicht ein bestimmtes Repertoire fest. Bildwerke konnten der Agora einen spezifisch politischen, aber auch einen allgemeiner >öffentlichen< Charakter unter Einschluss der agonalen Feste geben. Die Leute von Alabanda, die von Vitruv wegen ihrer sabwegigen< Praxis getadelt werden, hatten in Wirklichkeit ihre eigenen Parameter. Dasselbe gilt für alle anderen Räume des sozialen Lebens. Exemplarische Untersuchungen haben für römische Wohnhäuser die dichte Interferenz zwischen der Funktion der Räume und ihrer Ausstattung mit Mosaiken und Wandbildern aufgezeigt: Räume und Bilder bedingten sich - und interpretierten sich wechselseitig ${ }^{41}$.

- Soziale Zeit. Bildwerke waren auf soziale Situationen bezogen. So unterschieden sich etwa die Diskurse über die Perserkriege stark, je nach spezifischen Anlässen. An der Athener Agora war die Schlacht von Marathon um 460 v. Chr. in einem Gemälde in der Stoa Poikile, der >Bunten Halle , dargestellt, in dem die Protagonisten die Qualitäten der militärischen Führung, der tollkühnen Einsatzbereitschaft und des Selbstopfers für den Staat zur Schau stellten: Im öffentlichen Zentrum waren die heldenhaften und patriotischen Aspekte des Sieges gefragt. Kurz zuvor war auf der Bühne des Dionysos-Theaters Aischylos' Drama »Die Perser« aufgeführt worden, in dem der griechische Sieg einen Diskurs über Hybris und Untergang auslöst: Die Tragödie war ein Ort der komplexen Reflexion auf fundamentale ethische Kategorien menschlichen Handelns. Und zur selben Zeit konnten die Teilnehmer eines Symposions über das Bild auf einer Weinkanne lachen: Hier ist die Demütigung der Perser in einen obszönen homosexuellen Missbrauch eines Orientalen umgesetzt, der den Namen Eurymedon trägt, den Ort der letzten siegreichen Schlacht gegen ein persisches Heer ${ }^{42}$. Bildwerke waren stark bedingt von den sozialen Situationen, für die sie gemacht waren und in denen sie benutzt wurden, und sie trugen ihrerseits wirkungsvoll zu dem mentalen Klima dieser Situationen bei.

- Soziale Handlungen. Bildwerke waren Faktoren menschlicher Handlungen. Einerseits waren sie Objekte von Handlungen: in religiösen Ritualen oder politischen Debatten. Andererseits konnten sie selbst in die Angelegenheiten der Menschen eingreifen. Bildwerke sprechen durch ihre Inschriften die Betrachter an, etwa Grabstatuen, die Trauer oder Bewunderung für die Verstorbenen einfordern: »Bleib stehen und trauere beim Grab des schönen Kleoitas, wie schön er war und doch sterben musste«. In der römischen Kaiserzeit waren Bildwerke des regierenden Kaisers im ganzen Reich

41 In diesem Sinn grundlegende, theoretisch fundierte Untersuchungen zur Ausstattung von Privathäusern mit Mosaiken und Wandbildern: Muth 1998; Lorenz 2008.

42 Gemälde der Schlacht von Marathon: T. Hölscher 1974, 50-84; De Angelis 1996. - Aischyl.Pers.: Grethlein 2010, 74-104. - Perser-Kanne Hamburg: Schauenburg 1975; Wannagat 2001; Miller 2010. 
in den Räumen des politischen Lebens, der Verwaltungsgeschäfte und der Rechtsprechung gegenwärtig; hier diente die >Präsenz‘ des Herrschers dazu, den Handlungen der Administration und der Jurisdiktion kaiserliche Autorität zu verschaffen ${ }^{43}$.

\section{Der Status des Bildes und das Leben mit Bildern}

Welcher Status kommt unter diesen Umständen dem Bildwerk und welche Rolle dem >Betrachter zu? Die Funktion von Bildwerken, deren Herstellung und Wahrnehmung sowie der Umgang mit ihnen, hatten ihre Orte in konkreten Situationen des sozialen Lebens. Dieser Umstand prägt die gesamte Haltung und Einstellung zu den Bildwerken in der vormodernen Zeit.

Der neuzeitliche >museale Habitus ist von der Autonomie einer ästhetischen Sphäre geprägt: Das Museum als autonomer ästhetischer Raum, ausgegrenzt aus allen anderen sozialen Kontexten; das Werk der >Kunst< als autonomes ästhetisches Produkt, herausgelöst aus allen anderen Funktionen; der >Künstler< als autonomer Schöpfer und der Besucher als autonomer Betrachter, beide losgelöst aus allen sozialen Bindungen und Verpflichtungen. Die ästhetischen Handlungen, die mit dieser Vorstellung von >Kunst< verbunden sind, werden durch ein hohes Maß an kommunikativer Intensität geprägt: Der >Künstler<, der mit höchster kreativer Kraft ein Werk seiner subjektiven Vorstellung schafft; das Werk, das eine intensive Botschaft übermittelt; und der Betrachter, der das Werk mit eben so starker Intensität wahrnimmt und interpretiert und es dabei seinem eigenen kulturellen und mentalen Horizont einfügt. Der theoretische Rahmen, der dieser Situation entspricht, ist ein semiotisches Konzept der intensiven Kommunikation zwischen $>$ Sender $<$ und $>$ Empfänger<, Autor und Publikum.

Es ist offensichtlich, wie grundverschieden die Situation im antiken Griechenland und Rom war. Und dieser Unterschied betrifft nicht nur die Funktionen der Bildwerke, sondern das ganze Feld der Produktion und Rezeption von Bildkunst und dazu die Begriffe, mit denen wir uns den antiken Bildwerken zu nähern versuchen. >Museum < und >Betrachter<, $>$ Kreativität< der >Schöpfung $<$ wie der >Interpretation<, selbst >Kunst< und >Künstler<: All das gab es in der Antike nicht - zumindest nicht in dem Sinn, den wir damit verbinden.

Der ontologische Status des Bildwerkes war in der Antike nicht: eine intensive Botschaft von einem intensiven >Sender< an einen intensiven >Empfänger zu übermitteln, sondern: >da< zu sein, >präsent< zu sein im Kontext des sozialen Lebens.

Dem entsprechend war die Grundhaltung gegenüber Bildwerken nicht: sie mit exklusiver Intensität zu betrachten und zu interpretieren, sondern: mit den Bildwerken $z u$ leben und an ihrer Sphäre teilzuhaben.

43 Grabepigramm des Kleoitas: Peek 1955, Nr. 1223; Jeffery 1962, 197 n. 67. - Präsenz der römischen Kaiser in ihren Statuen: Pekáry 1985, 43. 
Damit soll gewiss nicht das grundsätzliche kommunikative Modell einer Konstellation von Sendern und Empfängern kultureller Zeichen geleugnet und auch nicht die Bedeutung der Rezeption von Bildwerken durch Individuen oder soziale Gruppen gemindert werden. Worum es geht, ist: dem >Hersteller und dem >Wahrnehmer $<$ der Bildwerke eine andere Rolle zuzuweisen - und damit auch dem Bildwerk selbst als Träger von Bedeutung einen anderen Status zuzuerkennen. Der >Hersteller< von Bildwerken ist kein >Schöpfer< einer Vorstellung, die ihren Sinn in sich selbst trägt und nach eigenen formalen Gesetzen entwickelt. Ebenso ist der >Wahrnehmer nicht ein intensiver Betrachter im Sinn des >musealen Habitus $<$. Auch der beliebte Begriff des »flüchtigen Betrachters«, der nur eben einmal unaufmerksam hinschaut, geht an der Sache vorbei, denn der Begriff »Betrachtung « reduziert den Umgang mit dem Bildwerk auf die reine Perzeption, und der Begriff der »Flüchtigkeit« suggeriert ein Defizit gegenüber dem Postulat einer adäquat intensiven Betrachtung.

Worum es geht, ist: >Partizipation< an einer gemeinsamen kulturellen >Welt<, im umfassenden Sinn, in dem man mit bedeutungsvollen Wesen der Lebenswelt zusammenlebt. Diese Welt umfasst mehr als den Kreis der Lebenden, die in der vorgegebenen Kontingenz des Lebens anwesend sind, zu ihr gehören auch die Götter und die Heroen der >mythischen< Vorzeit, die verstorbenen Vorfahren der jüngeren Vergangenheit und die nicht präsenten bedeutungsvollen Gestalten der Gegenwart mit ihren exemplarischen Eigenschaften und Leistungen. Die Bildhauer, Maler und anderen Handwerker geben diesen Gestalten konkrete bildhafte Präsenz im Leben der Gesellschaft. »Partizipation« bedeutet Teilhabe an gesellschaftlichen Situationen, in denen Bildwerke - neben anderen Elementen - eine mehr oder minder bedeutungsvolle Rolle spielten: bei religiösen Festen, Volksversammlungen, juristischen Angelegenheiten und Handelsgeschäften auf der Agora, Begräbnisritualen, Hochzeitszeremonien, privaten Ess- und Trinkgelagen und so fort. Hier fand >Leben mit Bildwerken< statt - das heißt: mit den dargestellten Gestalten und Vorgängen in ihrer >konzeptuellen Präsenzく.

Der Umgang mit den Bildwerken wurde in solchen Situationen zum Teil in rituellen Formen geregelt. Kult-Statuen wurden mit angemessenen Riten geweiht, verehrt und gepflegt, Weihgeschenke wurden nach traditionellen Sitten aufgestellt, erhalten und entsorgt, Ehrenstatuen wurden nach strikten Gebräuchen und sogar bindenden Gesetzen beschlossen und errichtet. Diese Regulierungen sind grundlegend für ein richtiges Verständnis der Bildwerke als Elemente des sozialen Lebens.

Normalerweise aber müssen Bildwerke bei diesem Umgang in derselben Weise wahrgenommen worden sein wie andere Elemente der sozialen Räume und Situationen. In der Regel waren die Menschen ja mit anderen Dingen beschäftigt: mit den Entscheidungen in den Volksversammlungen, den Rechtsfällen der Gerichtshöfe, den Handelsgeschäften, den Ritualen des Götterkults und der Totenverehrung und so fort. Dabei konkurrierten die Bilder mit anderen Personen und Gegenständen um Aufmerksamkeit. Die lebenden Menschen konnten ihnen starke, aber auch nur schwache Beachtung schenken. 
Auf der einen Seite konnten die Bildwerke stärksten Eindruck und höchste Bewunderung erregen. Das Standbild des Theagenes ist für die Bürger von Thasos über lange Zeit zum kollektiven Schicksal geworden; der Anführer der Männer in Aristophanes' »Lysistrate « nimmt das Bild des Aristogeiton als exemplarisches Verhaltensmuster in den eigenen Körper auf: Beides sind Zeugnisse für höchst intensives Sehen. Und noch Jahrhunderte später wünscht sich Asinius Pollio, dass die Besucher seines Wohnsitzes die dort aufgestellten Bildwerke »vehementer« anschauen ${ }^{44}$. Bei diesem intensiven Sehen spielten auch die formalen, stilistischen Aspekte der Bildwerke eine große Rolle: Sie waren es ja, die der >Präsenzく der Gestalten und Vorgänge ihre Evidenz und Wirkung gaben. Hier liegt die Wurzel für ästhetische Diskurse, Reflexionen und Theorien zur antiken Bildkunst, die seit der klassischen Zeit Griechenlands bis in die römische Kaiserzeit zu einer gewissen Komplexität geführt wurden. Allerdings blieben diese theoretischen Diskurse in der Regel - explizit oder implizit - den Themen und Funktionen der Bildwerke untergeordnet: Der Stil war nicht ein autonomer Ausdruck einer individuellen schöpferischen Kreativität, sondern in der formalen Gestaltung wurden die Qualitäten und Aspekte zum Ausdruck gebracht, um derentwillen die Bildgegenstände, d. h. die dargestellten Gestalten und Vorgänge, >Bedeutung im Lebenskontext der Gesellschaft erhalten sollten. Museen im modernen Sinn, als Räume einer reinen Betrachtung von >Kunst<, gab es nicht.

Auf der anderen Seite aber muss die Wahrnehmung von Bildwerken oft sehr viel weniger intensiv gewesen sein: Die Menschen konnten sie einfach als Gegebenheit hinnehmen, sie tolerieren, sie nur gelegentlich bemerken oder sie auch völlig ignorieren. Kurzum: Sie müssen sich zu den Bildwerken wie zu anderen Personen und Gegenständen des Lebens verhalten haben. Partizipation findet in Akten von mehr oder minder starker Aufmerksamkeit statt. Die Aufmerksamkeit wird von den Situationen und Diskursen der Gesellschaft und ihrer Individuen gesteuert. Dabei konnten sie den Gestalten und Vorgängen der Bilder größere oder kleinere Bedeutung beimessen. Nur selten allerdings werden die Menschen in der Antike die Bildwerke in unserem Sinn intensiv, d. h. >um ihrer selbst willen studiert $<$ und interpretiert haben.

\subsection{Dichte Bedeutung und schlechte Sichtbarkeit}

Dies alles hat auch Folgen für die Sichtbarkeit der Bildwerke, die von einer eigentümlichen Ambivalenz geprägt ist. Die Standbilder von Athleten und Staatsmännern waren in den öffentlichen Räumen so aufgestellt, dass sie gut sichtbar waren für alle, die sie sehen wollten und sollten: Hier führte die kompetitive Situation des Strebens nach öffentlichem Ansehen zu einer Aufstellung in den Brennpunkten des Lebens. Auch die Standbilder der Götter und Heroen, ebenso wie die Vielzahl der Votivbilder in den Heiligtümern und 
nicht zuletzt die Statuen und Reliefs der Verstorbenen auf den Gräbern, wurden wegen ihrer exemplarischen Bedeutung nahsichtig für die Lebenden positioniert. Das >Leben mit< diesen Bildwerken war >auf Augenhöhe< möglich.

Daneben aber gab es viele Bildwerke, die erstaunlich wenig Rücksicht auf Sichtbarkeit nahmen. Das ist für unser Verständnis von >Kunst< als visueller Botschaft irritierend und die Forschung ist diesem Phänomen darum auch gewöhnlich ausgewichen. Ein Verständnis dessen, was Bildkunst in Griechenland war, ist jedoch nur möglich, wenn wir uns diesem Phänomen stellen 45 .

Das Schatzhaus von >Sikyon<, das vermutlich auf allen Seiten mit Metopen geschmückt war, kann in Delphi bestenfalls von drei Seiten sichtbar gewesen sein. Das Schatzhaus von Siphnos jedenfalls war an dem Prozessionsweg so positioniert, dass man es zunächst von der Rückseite sah, dann die eine Längsseite passierte, sich schließlich zur Frontseite umwenden musste - und die abgewandte Längsseite überhaupt nicht sehen konnte. Dasselbe gilt sogar für das politisch anspruchsvolle Schatzhaus von Athen. Die >Bild-Programme< solcher Bauwerke stellten zwar eine komplexe Konstellation von Themen dar - diese konnten aber nur reduziert wahrgenommen werden.

Dies Paradox zeigt sich in aller Klarheit an dem größten und komplexesten Ensemble der griechischen Bildkunst, dem Parthenon ${ }^{46}$. Wer sich dem Bau näherte, blickte wahrscheinlich zuerst auf die großen Giebelkompositionen, die Athena als Herrin des Tempels in den Vordergrund stellten: im Osten ihre Geburt im Kreis der Götter, im Westen ihren Sieg über Poseidon im Wettstreit um die Herrschaft über Athen. Um diese Beziehung der beiden Themen zueinander zu realisieren, muss man sich zunächst von einer Fassade zur anderen bewegen. Schwieriger wird es schon bei den Metopen, die sich um alle vier Seiten ziehen und einen kohärenten Zyklus von Mythen schildern: Die Götter im Kampf gegen die Giganten, die heroischen Lapithen gegen die Kentauren, die Athener gegen die Amazonen und die griechischen Helden gegen Troia. Sie ergänzen einander gegenseitig zu einer hochbedeutsamen Sequenz von archetypischen Kämpfen griechischer Götter und Heroen gegen Feinde der griechischen Kultur. Dieser Zyklus erschließt sich nur beim völligen Umkreisen des Baues. Mit noch größeren Schwierigkeiten war der Fries zu betrachten, der sich innerhalb des Säulenkranzes um die Cella zog. Er schilderte mit vielen Details die große Prozession der athenischen Bürgerschaft zum Fest der Athena. Wer das verfolgen wollte, musste noch einmal neu ansetzen, musste an der Ecke im Südwesten anfangen, den Tempel zunächst im Westen und Norden bis zum Ziel des Zuges im Zentrum der Ostseite umschreiten, dann vom selben Ausgangspunkt noch einmal die Südseite passieren bis zur Ostseite, um zu begreifen, dass die Prozession den Bau auf beiden Seiten in zwei parallelen Zughälften umschritt. Es ist schwer denkbar, dass viele Besucher der Akropolis diese Folge von komplexen Bewegungen vollzogen haben.

45 Zum Folgenden s. T. Hölscher 2009, 54-57.

46 Zum Folgenden s. T. Hölscher 2009, 54-57. Unabhängig in ähnlichem Sinn: Marconi 2009. 
Der Fries war zudem nur in großer Höhe und im Halbdunkel des Umgangs zu betrachten, in den, anders als in der heutigen Ruine, kein Licht von oben fiel. Wer das Friesband in seiner Längserstreckung verfolgen wollte, musste in den Säulenumgang eintreten, wo aber der äußerst steile Sichtwinkel jede genauere Betrachtung verhinderte. Ein flacherer Ansichtswinkel ergab sich von außen, doch hier wurde die Folge der Prozession in kurzen Abständen von den Säulen unterbrochen. Sicher nicht erkennbar waren die vielen signifikanten Details. So ist die Kavalkade der verwirrend gestaffelten Reiter in zehn Gruppen gegliedert, die offenbar die zehn attischen Phylen darstellen; doch die subtilen Charakterisierungen durch Einzelheiten der Kleidung und Ausrüstung können unter den genannten Sichtbedingungen nicht unterscheidbar gewesen sein.

Überhaupt war der bildliche Schmuck griechischer Architektur außerordentlich Betrachter-unfreundlich. Das gilt insbesondere für die zentralen Bauten griechischer Städte: die Tempel. Hier waren Bildwerke entweder in den monotonen Reihen der Metopen oder in den endlosen schmalen Friesen oder schließlich in den flachen Dreiecken der Giebel angebracht. All diese Bildformate sind denkbar schlecht geeignet, um wichtige Bildthemen wirkungsvoll einem Betrachter vor Augen zu führen. Darüber hinaus sind die Bilder in großer Höhe angebracht, was einen Blick aus weiter Entfernung und/oder aus steilem Winkel bedingt.

Keine Frage also, dass all die vielen bedeutungsvollen Details kaum wahrzunehmen waren, vor allem während der religiösen Rituale, die die Aufmerksamkeit weitgehend anderweitig beansprucht haben müssten.

Hier wird ein Grundzug griechischer Bildkunst deutlich, den man auch sonst immer wieder antrifft. Viele Bildwerke der griechischen Kunst nehmen erstaunlich wenig Rücksicht auf den Betrachter. Die schönsten griechischen Vasen wurden den Toten in die Gräber mitgegeben, wo sie niemand mehr zu sehen bekam. Wertvolle Gemmen mit eingeschnittenen Bildern wurden in Ringen am Finger getragen, wo sie sich, bei ständiger Bewegung der Hand und in ihrem Miniaturformat, keinem normalen Blick darboten. Und noch das größte politische Denkmal Roms, die Traianssäule, entfaltet ihren vielszenigen Bildbericht der Kriege Traians gegen die Daker in einer Höhe und in einer steilen Ansicht, in der kein menschliches Auge die höchst komplexen politischen Aussagen wahrnehmen konnte. An die gotischen Kathedralen mit ihrem Bildschmuck in großer Höhe muss nur >im Vorbeigehen< erinnert werden.

Was sagt dies Paradox über die Bildwerke aus? Und was über die Formen, mit diesen Bildwerken zu leben? Auch hier ist das Konzept des >Lebens mit Bildern< hilfreich.

Der griechische Begriff für architektonischen Bildschmuck war »kosmos«. Dieser Begriff bezeichnet Schmuck, zugleich aber auch die richtige, angemessene, sinnvolle Ordnung, die die Bild- und Bauwerke miteinander ergeben ${ }^{47}$. Man kann von hier aus 
die Brücke zu den lateinischen Begriffen »ornamentum« und »decor« schlagen, die die Grundlage einer fruchtbaren, weit mehr als ästhetischen Theorie bilden. Das kann hier nur knapp skizziert werden.

Das Schatzhaus von >Sikyon< wie der Parthenon tragen reichen Bildschmuck und sind zugleich reiche, geordnete Bilderwelten. Dasselbe gilt auch für die Ausstattung von Lebensräumen mit Bildern: Auf der Agora bildeten die Standbilder von Staatsmännern, mythischen Heroen und Göttern eine ideale Ordnung des Gemeinwesens; in den Heiligtümern schlossen sich die geweihten Bildwerke zu einem Sinngeflecht religiöser Vorstellungen zusammen; in den Nekropolen stellten Bilder die soziale Welt der Verstorbenen in den Rahmen von Leben und Tod. Überall entstanden bildhafte Konzepte eines Kosmos, die zugleich >Schmuck $<$ und >Ordnung $<$ sind.

Diese Ordnungen aber hatten zu einem gewissen Grad ihr eigenes Leben, unabhängig von den Menschen, die in ihnen lebten. Die Götter, mythischen Heroen und berühmten Menschen, die in den Bildwerken gegenwärtig gehalten wurden, entfalteten ihre primäre Wirkung zunächst durch ihre reine Präsenz.

Weil die Gestalten der Bildkunst so unmittelbar präsent waren, besaßen sie eine gewisse Autonomie gegenüber dem Betrachter. Sie >sind da< und stellen eine >Bilder-Welt< dar, auch wenn die lebenden Menschen sie nur eingeschränkt oder gar nicht sehen konnten bzw. sie nur eingeschränkt wahrnahmen oder gar ignorierten. Die Menschen waren nicht ständig mit dem intensiven Betrachten, Verstehen und Interpretieren der Bildwerke beschäftigt. Sie >lebten $<$ mit den Bildern.

Die Bildwerke waren mehr als zielgerichtete Botschaften von bestimmten Autoren/ Sendern an bestimmte Empfänger. Sie bildeten Welten von sinnvollen Bildern, in denen die Menschen ihr Leben einrichten und von denen sie ihre Maßstäbe des Lebens ableiten konnten. Der Kosmos der antiken Bildwerke ist mehr als ihre Wirkung auf den Betrachter: Er ist die Ordnung des Lebens selbst.

\section{Bibliographie}

\section{Amandry 1953}

P. Amandry, La colonne des Naxiens et le portique des Athéniens. FdDelphes II (Paris 1953).

\section{Amandry 1957}

P. Amandry, A propos de Polyclète: Statues de Olympioniques et carriere de sculpteurs, Charites. Studien zur Altertumswissenschaft. Festschrift Ernst Langlotz (Bonn 1957) $63-87$.

\section{Bettinetti 200I}

S. Bettinetti, La statua di culto nella pratica rituale greca (Bari 2001). 


\section{Bielefeld I962}

E. Bielefeld, Bronzestatuette des Wadsworth Athenaeum in Hartford, Connecticut, AntPl 1 (Berlin 1962) 39-41 Taf. 38-44.

\section{Bielfeldt 2012}

R. Bielfeldt, Polis Made Manifest. The Physiognomy of the Public in the Hellenistic City with a Case Study of the Agora in Priene, in: C. Kuhn (Hg.), Politische Kommunikation und öffentliche Meinung in der antiken Welt (Stuttgart 2012) 87-122.

Bol 2002

P. C. Bol (Hg.), Die Geschichte der antiken Bildhauerkunst I (Mainz 2002).

\section{Brunnsåker I97I}

S. Brunnsåker, The Tyrant-Slayers of Kritios and Nesiotes ${ }^{2}$ (Stockholm 1971) [Orig. 1953].

\section{Chaniotis in Vorbereitung}

A. Chaniotis, Emotional Responses to Statues (in Vorbereitung).

De Angelis 1996

F. De Angelis, La battaglia di Maratona nella Stoa Poikile, AnnPisa 1, 1996, 119-171.

\section{Dillon 2006}

S. Dillon, Ancient Greek Portrait Sculpture (Cambridge 2006).

\section{Fehr 1984}

B. Fehr, Die Tyrannentöter oder: Kann man der Demokratie ein Denkmal setzen? (Frankfurt a. M. 1984).

\section{Freyer-Schauenburg 1974}

B. Freyer-Schauenburg, Bildwerke der archaischen Zeit und des Strengen Stils, Samos XI (Bonn 1974).

\section{Gell 1998}

A. Gell, Art and Agency. An Anthropological Theory (Oxford 1998).

\section{Grandjean - Salviat 2000}

Y. Grandjean - F. Salviat, Guide de Thasos (Athènes 2000).

\section{Grethlein 2010}

J. Grethlein, The Greeks and Their Past (2010).

\section{Gross 1969}

W. H. Gross, Quas iconicas vocant. Zum Porträtcharakter der Statuen dreimaliger olympischer Sieger, NAWG, 1969, 3 . 


\section{Gruben 200I}

G. Gruben, Tempel und Heiligtümer der Griechen ${ }^{5}$ (München 2001) [Orig. 1966].

\section{Herrmann 1988}

H.-V. Herrmann, Die Siegerstatuen von Olympia, Nikephoros 1, 1988, 119-183.

\section{Himmelmann 1990}

N. Himmelmann, Ideale Nacktheit in der griechischen Kunst (Berlin 1990).

\section{Himmelmann 200I}

N. Himmelmann, Die private Bildnisweihung bei den Griechen (Wiesbaden 2001).

\section{F. Hölscher 2004}

F. Hölscher, Rez. S. Bettinetti, La statua di culto nella pratica rituale greca (Bari 2001), Gnomon 77, 2004, 433-437.

\section{F. Hölscher 2005}

F. Hölscher, Kultbild, Thesaurus cultus et rituum antiquorum IV, 2005, 52-65.

\section{F. Hölscher 2010}

F. Hölscher, Die Tyrannenmörder - ein Denkmal der Demokratie, in: E. SteinHölkeskamp - K.-J. Hölkeskamp (Hgg.), Die griechische Welt. Erinnerungsorte der Antike (München 2010) 244-258.

\section{T. Hölscher 1974}

T. Hölscher, Griechische Historienbilder des 5. und 4. Jahrhunderts v. Chr. (Würzburg 1974).

\section{T. Hölscher 2002}

T. Hölscher, Rituelle Räume und politische Denkmäler im Heiligtum von Olympia, in: H. Kyrieleis (Hg.), Olympia 1875 - 2000. 125 Jahre deutsche Ausgrabungen, Internationales Symposion, Berlin, 9.-11. November 2000 (Mainz 2002) 331-345.

\section{T. Hölscher 2007}

T. Hölscher, Die griechische Kunst, Beck Wissen 2551 (München 2007).

\section{T. Hölscher 2009}

T. Hölscher, Architectural Sculptures: Messages? Programs? Towards Rehabilitating the Notion of >decoration««, in: P. Schultz - R. von den Hoff (Hgg.), Structure, Image, Ornament. Architectural Sculpture in the Greek World (Oxford 2009).

\section{Jeffery 1962}

L. H. Jeffery, The Inscribed Gravestones of Archaic Attica, BSA 57, 1962, 115-157. 


\section{Keesling 2003}

C. Keesling, The Votive Statues of the Athenian Acropolis (Cambridge 2003).

\section{Krumeich 1997}

R. Krumeich, Bildnisse griechischer Herrscher und Staatsmänner im 5. Jahrhundert v. Chr. (München 1997).

\section{Knell 1990}

H. Knell, Mythos und Polis. Bildprogramme griechischer Bauskulptur (Darmstadt 1990).

\section{Lorenz 2008}

K. Lorenz, Bilder machen Räume (Berlin 2008).

\section{Mann 200I}

C. Mann, Athlet und Polis im archaischen und frühklassischen Griechenland (Göttingen 2001).

\section{Marconi 2004}

C. Marconi, Kosmos. The Imagery of the Archaic Greek Temple, Res: Anthropology and Aesthetic, 2004, 209-224.

\section{Marconi 2009}

C. Marconi, The Parthenon Frieze. Degrees of Visibility, Res: Anthropology and Aesthetics 55/56, 2009, 156-173.

\section{Meier 1980}

C. Meier, Die Entstehung des Politischen bei den Griechen (Frankfurt a. M. 1980).

\section{Miller 20I0}

M. C. Miller, I Am Eurymedon. Tensions and Ambiguities in Athenian War Imagery, in: D. M. Pritchard (Hg.), War, Democracy and Culture in Classical Athens (Cambridge 2010) 304-338.

\section{Morris 1992}

S. Morris, Daidalos and the Origins of Greek Art (Princeton 1992).

\section{Olmos 1992}

R. Olmos, El amor del hombre con la estatua. De la Antiguedad a la Edad Media, in: H. Froning - T. Hölscher - H. Mielsch, Kotinos. Festschrift Erika Simon (Mainz 1992) $256-266$.

\section{Osborne - Tanner 2007}

R. Osborne - J. Tanner (Hgg.), Art's Agency and Art History (Oxford 2007). 


\section{Muth 1998}

S. Muth, Erleben von Raum - Leben im Raum. Zur Funktion mythologischer Mosaikbilder in der römisch-kaiserzeitlichen Wohnarchitektur, Archäologie und Geschichte 10 (Heidelberg 1998).

\section{Niemeyer 1996}

H. G. Niemeyer, SEMATA. Über den Sinn griechischer Standbilder (Hamburg 1996).

\section{Pandermalis 1969}

D. Pandermalis, Untersuchungen zu den klassischen Strategenköpfen (Freiburg i. Br. 1969).

\section{Peek I955}

W. Peek, Griechische Vers-Inschriften (Berlin 1955).

\section{Pékary 1985}

T. Pekáry, Das römische Kaiserbildnis in Staat, Kult und Gesellschaft, dargestellt anhand der Schriftquellen, Das römische Herrscherbild 3 (Berlin 1985).

\section{Raubitschek 1939}
A. E. Raubitschek, Leagros, Hesperia 8, 1939, 155-164.

\section{Raubitschek 1949}

A. E. Raubitschek, Dedications from the Athenian Acropolis (Cambridge, Mass. 1949).

\section{Rausa I994}

F. Rausa, L’immagine del vincitore (Treviso 1994).

\section{Richter 1965}

G. M. A. Richter, The Portraits of the Greeks I-III (London 1965).

\section{Rolley 1994}

C. Rolley, La sculpture grecque (Paris 1994).

\section{Schauenburg 1975}

K. Schauenburg, Eurymedon eimi, AM 90, 1975, 97-121.

\section{Scott 2010}

M. Scott, Delphi and Olympia. The Spatial Politics of Panhellenism in the Archaic and Classical Periods (Cambridge 2010).

\section{Scheer 2000}

T. S. Scheer, Die Gottheit und ihr Bild (München 2000).

\section{Simon I988}

E. Simon, Eirene und Pax. Friedensgöttinnen in der Antike (Stuttgart 1988). 


\section{Smith 2007}

R. R. R. Smith, Pindar, Athletes and the Early Greek statue habit, in: C. Morgan -

S. Hornblower (Hgg.), Pindar's Patrons, Poetry and Festival (Oxford 2007) 83-139.

\section{Stemmer 1995}

K. Stemmer (Hg.), Standorte. Kontext und Funktion antiker Skulptur.

Ausstellungskatalog Berlin (Berlin 1995).

Steiner 200I

D. Steiner, Images in Mind (Princeton 2001).

\section{Stürner 2005}

R. Stürner, Europäische Medienfreiheit und nationale Medienkultur, Jahrbuch der Heidelberger Akademie der Wissenschaften, 2005, 71-73.

\section{Tanner 1992}

J. J. Tanner, Art as Expressive Symbolism. Civic Portraits in Classical Athens, CambrAJ 2, 1992, 167-190.

\section{Tanner 2006}

J. J. Tanner, The Invention of Art History in Ancient Greece (Oxford 2006).

\section{Taylor 1992}

M. W. Taylor, The Tyrant-Slayers (Salem 1998).

Thompson - Wycherley 1972

The Agora of Athens, Agora XIV (Athens 1974).

\section{Wannagat 200I}

D. Wannagat, >Eurymedon eini<. Zeichen ethnischer, sozialer und ohysischer Differenz in der Vasenmalerei des 5. Jh., in: R. von den Hoff - S. Schmidt (Hgg.), Konstruktionen von Wirklichkeit (Stuttgart 2001) 51-71.

\section{Wycherley 1957}

R. E. Wycherley, Literary and Epigraphical Testimonia. Agora III (Princeton, N. J. 1957).

\section{Zanker 1995}

P. Zanker, Die Maske des Sokrates (München 1995). 